\title{
A Control System for MRI-Guided Conformal Interstitial Thermal Therapy
}

\author{
R. Chopra, S.N. Baker, M. Burtnyk, A.J. Weymouth, and M.J. Bronskill \\ Sunnybrook and Women's College Health Sciences Centre, Toronto, Canada, M4N3M5 \\ rajiv.chopra@utoronto.ca
}

\begin{abstract}
A control system has been developed to perform MRI-guided interstitial ultrasound heating in a closed-bore MR scanner. A prototype delivery system capable of positioning, rotating, and delivering RF power to a heating applicator has been built and algorithms for using temperature information as a source of feedback for the control system have been tested. Heating experiments indicate that the system is capable of producing repeatable thermal lesions. Simulations of heating with the control algorithm enable the automatic selection of parameters to achieve a conformal region of thermal coagulation.
\end{abstract}

\section{Introduction}

Interstitial thermal therapy involves the delivery of energy from small $(<5 \mathrm{~mm})$, invasive devices inserted directly within or adjacent to a target volume in tissue. The goal of this form of therapy is to heat a target volume of tissue to temperatures sufficient to produce thermal coagulation, typically above $60^{\circ} \mathrm{C}$. [1]. A challenge with interstitial thermal therapy is to deliver sufficient energy to coagulate the entire target volume, while sparing surrounding normal tissue from unwanted damage.

Visual access to the target site is not available in interstitial thermal therapy; rather, the procedure relies on information provided by imaging technology to verify and control the treatment. Imaging information is preferable throughout the treatment; to guide the heating applicators to the correct location, to monitor the progression of the heating pattern, and to evaluate the extent of thermal damage upon completion. MRIguided interstitial thermal therapy combines the capability of MRI to produce volumetric measurements of the structure, function, temperature and viability of tissue with heating devices capable of controlling the spatial deposition of energy. The integration of these technologies can improve the ability of interstitial heating to coagulate tissue volumes that conform to the geometry of a target.

Our group is developing multi-frequency interstitial ultrasound heating applicators for use in MRI-guided conformal thermal therapy. These devices incorporate planar transducers with self-collimated acoustic fields that confine thermal coagulation to angular sectors of tissue through device rotation [2]. The adjustment of device parameters such as rotation rate, power and frequency provide the ability to vary the geometry of the thermal lesion during heating. 
The capability to adjust multiple parameters with these applicators enables control over the spatial heating pattern, but also results in the requirement for a more complex delivery system. Along with this level of complexity comes the capability to adjust parameters during heating to shape the thermal damage pattern. There are two parameters that can be adjusted for each element (power and frequency) as a function of the angular position of the device. The rotation rate of the applicator is also adjustable, resulting in a total of $2 n+1$ angle-dependent parameters, where $n$ is the number of elements in the applicator.

The level of control required to execute a thermal therapy with a simple 2-element heating applicator is beyond the reasonable limit for manual operation; thus, another goal of this project is the development of a control system to automate the selection of exposure parameters. This work describes ongoing development of the hardware and software to control the heating applicator signals and motion to generate a conformal heating pattern in tissue.

\section{Methods/Materials}

\subsection{Interstitial Ultrasound Heating Applicators}

Dual-element interstitial ultrasound heating applicators have been built for investigational use in MRI-guided conformal thermal therapy. The applicators, shown in Figure 1, are constructed from MRI-compatible materials such that MR imaging can be performed in the vicinity of the device during treatment without significant artifact.

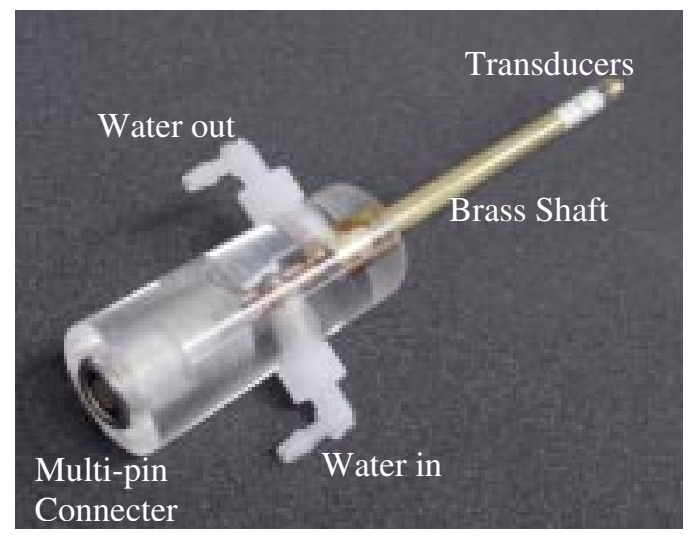

Fig. 1. Photograph of multi-element interstitial ultrasound heating applicators for MRI-guided thermal therapy.

Each applicator consists of two planar multi-frequency ultrasound transducers, $4 \times 10 \mathrm{~mm}$, with self-collimated acoustic beams that can be controlled independently. The resonant frequencies of the elements are typically 4.7 and 9.7 MHz. Electrical 
connections are made at the back of the applicator, and water flows through the device at a rate of $300 \mathrm{ml} / \mathrm{min}$ to remove heat from thermal losses in the transducers, and to couple sound from the elements into surrounding tissue.

\subsection{Treatment Delivery System}

The treatment delivery system for the heating applicators is on a mobile cart, shown in Figure 2, located adjacent to the operator console of the MRI during use. The system consists of a computer with a PCI-based signal generator (NI-5401,

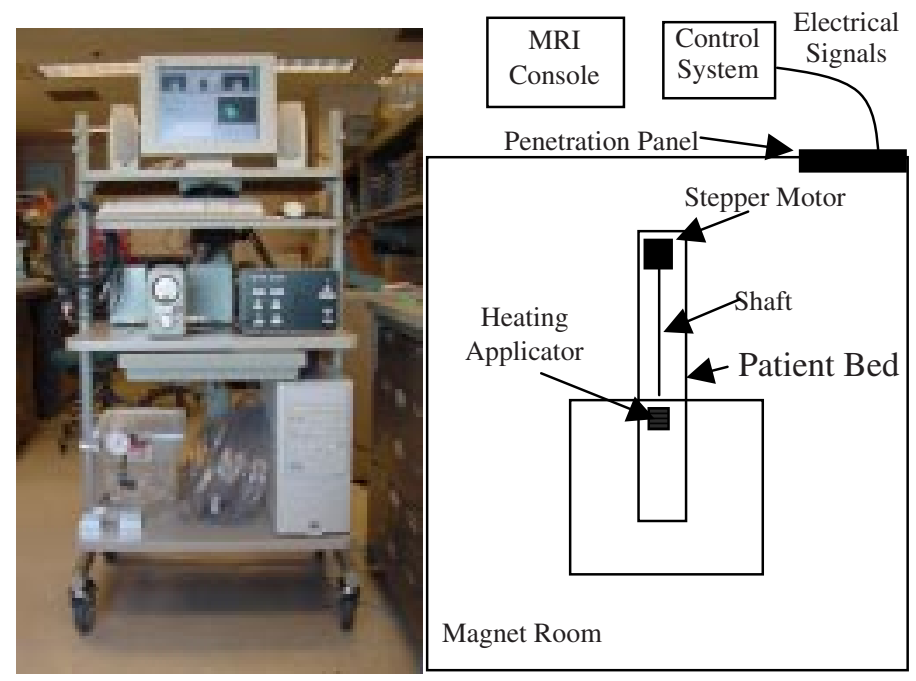

Fig. 2. Control system for MRI-guided thermal therapy with multi-frequency interstitial ultrasound heating applicators. The computer with the control software, the RF electronics, and the water flow equipment is all located on a mobile cart, shown in the left panel. The schematic location and setup of the control system and equipment is shown on the right panel.

National Instruments, USA) for each element in the heating applicator. The rotation of the heating applicator is currently controlled by a stepper motor driver and controller (NI-7334, MID-7602, National Instruments, USA). Each RF channel passes through a custom-built RF amplifier and matching network to deliver adequate power to heat tissue. Dual-directional couplers (Pulsar Microwave, USA) measure the net power delivered to the transducer continuously during heating An analog I/O card (NI-6024, National Instruments, USA) measures various system variables such as the net power delivered to the elements, as well as the pressure and flow rate of the water circuit.

The flow circuit delivers water to the heating applicator from a pump located on the cart, through approximately 50ft of flexible tubing passing into the MRI suite through the waveguide. The electrical cables to the transducer are passed through the grounded penetration panel into the magnet room. In the current experimental configuration, the stepper motor is located at the foot of the MRI bed, connected to the heating applicator via a long shaft. 
The synchronization and control of the various aspects of the delivery system are accomplished with software written in LabVIEW, which enables one to enter a sequence of parameters for a treatment as a function of rotation angle. The treatment plan is converted into the appropriate sequence of device rotation and RF signals, and displays the measured power, flow rate and pressure during execution. The execution of the treatment plan is done automatically, but the system design includes the possibility of adjustment during treatment based on temperature or other feedback from MR imaging.

\subsection{Temperature Based Control Algorithm}

MRI provides the capability to acquire high-resolution $(\sim \mathrm{mm})$ maps of the temperature distribution with a temporal resolution of a few seconds and temperature resolution of a few degrees. A control algorithm has been developed which uses the temperature information provided by MRI as a source of feedback for the control system. The temperature at the boundary of the target along the beam axis of the transducer is chosen as the control point. The rate of rotation, power, and frequency are chosen based on the difference between the temperature at the control point and the target temperature of $55^{\circ} \mathrm{C}$. The control algorithm also considers the maximum temperature along the beam direction, turning off the power if the temperature exceeds a critical temperature (e.g. $80^{\circ} \mathrm{C}$ ).

The control algorithm has been tested for a single element using a 3-dimensional numerical simulation of the heating process. The ultrasound field from the transducers is calculated using a Rayleigh-Sommerfeld integral [3]. The acoustic power distribution is calculated from the field, and is used as the source function for heating in the Bio-heat transfer equation [4]. A finite-difference algorithm is implemented to calculate the resulting temperature distribution as the heating applicator rotates about it axis. The spatial resolution in the calculations is $1 \mathrm{~mm}^{3}$ and the temporal resolution is 1 second.

\section{Results/Discussion}

Benchtop heating experiments have been carried out in tissue mimicking gels [5] to verify the repeatability and control over the production of thermal damage patterns with this delivery system. Heating applicators were inserted into a container with the gel material and rotated over $360^{\circ}$ with a constant frequency $(4.7 \mathrm{MHz})$ and power (10W) to each element. The scan rate was varied across the rotation from $5 \% \mathrm{~min}$ to $60 \%$ min in $30^{\circ}$ sectors (12 steps). The thermal damage pattern was recorded in the gel through the coagulation of a suspended protein, and measured with MR imaging as a consequence of a change in T2. Multiple experiments were performed to analyze the repeatability of the system to deliver this treatment.

A T2-weighted image through the heating pattern transverse to the location of the heating applicator is shown in the left panel of Figure 4. The coagulated gel material appears as a region of lower signal intensity due to the reduction of $\mathrm{T}_{2}$ induced by 
heating. The gradual reduction in the radius of thermal coagulation extending from the applicator due to the increase in scan rate is apparent from the figure. The initial position and direction of rotation of the applicator is also shown in the left panel. The right panel in Figure 4 shows a graph representing measurements of the radius (measured on T2-weighted images) of thermal coagulation as a function of applicator position for multiple experiments. The graph indicates a good level of repeatability in the execution of the delivery system and the performance of the heating applicators as measured by the shape of the heated volume.
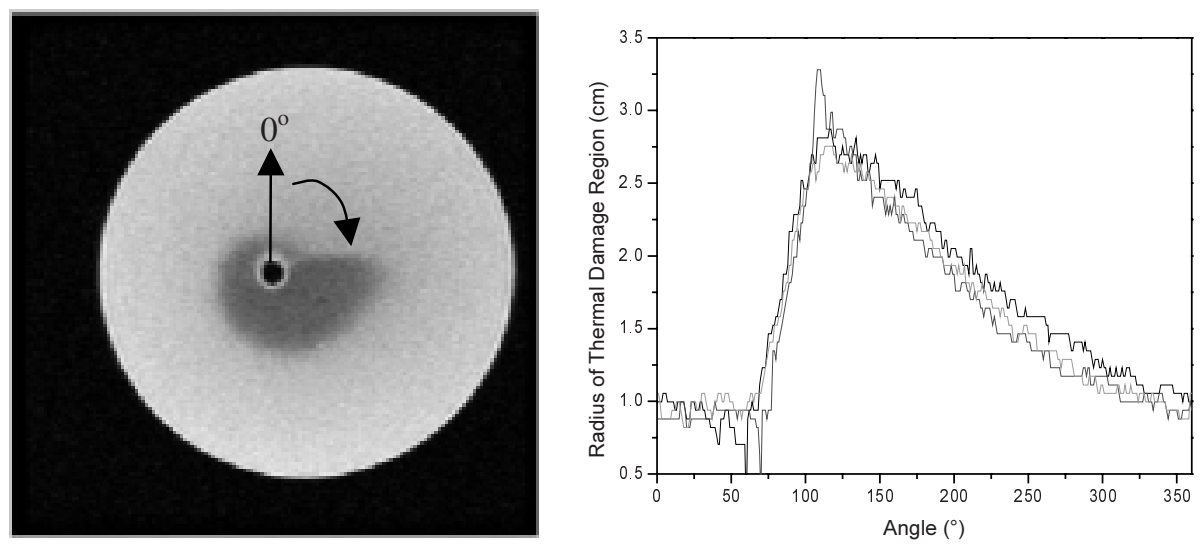

Fig. 3. (left) $\mathrm{T}_{2}$-weighted image transverse to the heating applicator after heating in a tissuemimicking gel material. The dark region is the thermally coagulated gel material with lower $\mathrm{T}_{2}$ than the surrounding gel. (right) Graph of the radius of thermal coagulation from three heating experiments such as the one shown in the left panel.

To test the control algorithm, a sample tumour boundary was obtained from an MR image, shown in Figure 4. A simulation was performed with the applicator at the centre of the tumour boundary. Values for tissue thermal and ultrasound parameters were chosen to match brain tissue, and perfusion was not included in the calculations. The control algorithm sampled the temperature at the control point were made every second, and the spatial resolution of the temperature maps was set to $1 \mathrm{~mm}$. The polar plot in Figure 4 shows contours representing the tumour boundary (black line), the region of thermal coagulation (temperatures $>55^{\circ} \mathrm{C}$, dashed gray line), and boundary beyond which no thermal damage occurs (thermal dose $<30$ eq. min, dotted gray line). The final graph in Figure 4 shows the error (in $\mathrm{mm}$ ) between the damage contours and the tumour boundary. The maximum error between the region of thermal coagulation and the tumour boundary is approximately $2 \mathrm{~mm}$. No thermal damage occurs beyond $9 \mathrm{~mm}$.

\section{Conclusions}

A prototype control system for interstitial ultrasound heating experiments has been designed and built for experiments in the MRI environment. Benchtop testing of the 
apparatus in a gel phantom indicates that the system is able to generate complex treatment patterns accurately and with repeatability. The control algorithm has been tested in simulations and has been shown to work well in adjusting thermal damage patterns to match target geometries.
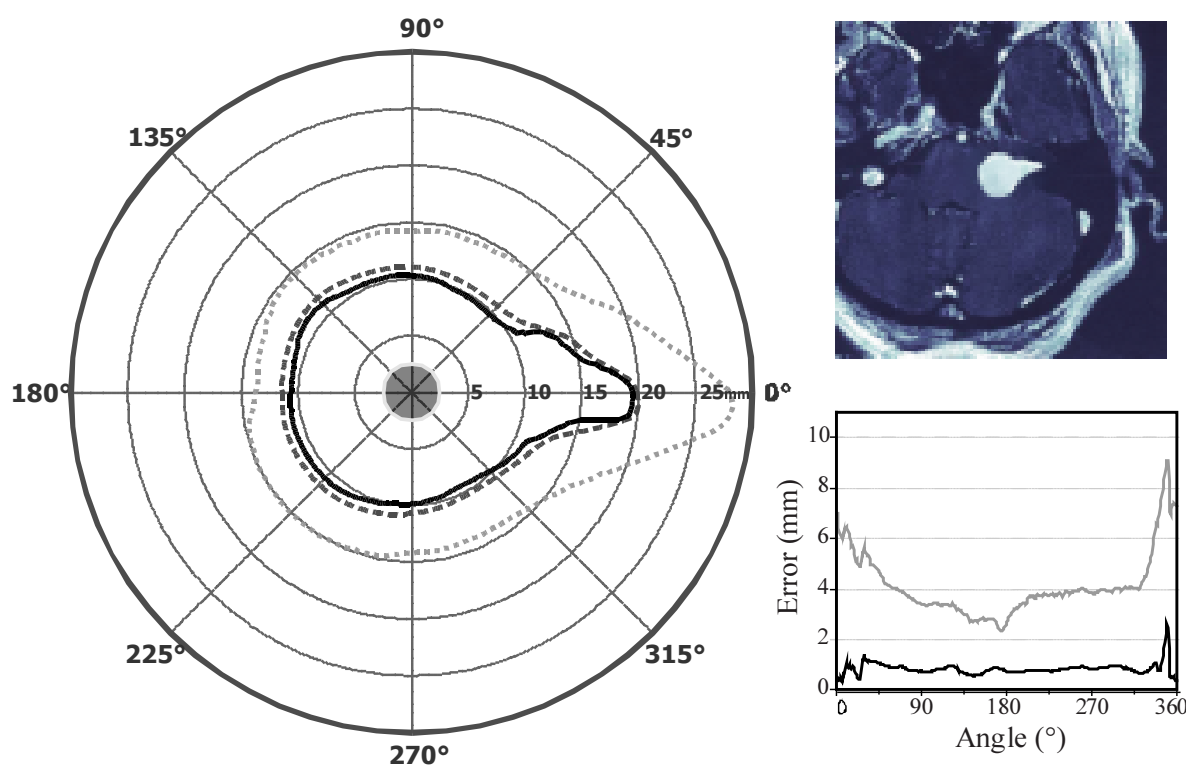

Fig. 4. Top right: MR image of a brain tumour used as a target volume for a simulated treatment using the ultrasound heating applicator with temperature feedback. Left: Polar plot of the tumour boundary (black) and the contours representing thermal coagulation (dark gray dash) and the boundary beyond which no thermal damage occurs (light gray dots). Bottom right: The error (in $\mathrm{mm}$ ) between to two damage contours and the tumour boundary.

The next stage in the development of this project is to use this control system to compare heating patterns with those predicted by numerical calculations. The verification of the performance of the control system in the MRI environment is also underway.

\section{References}

1. Thomsen, S.: Mapping of thermal injury in biologic tissues using quantitative pathologic techniques. Proc SPIE 3594 (1999) 82-952.

2. Chopra, R., Luginbuhl, C., Weymouth, A.J., Foster, F.S., Bronskill, M.J.: Interstitial ultrasound heating applicator for MR-guided thermal therapy. Phys Med Biol, 46 (2001) 3133-45

3. Ocheltree KB, Frizzell L.A.: Sound field calculation for rectangular sources. IEEE Trans Ultrason Ferroelect Freq Contr, 36 (1989) 242-248.

4. Pennes, H.H.: Analysis of tissue and arterial blood temperatures in the resting human forearm. J Appl Physiol, 1 (1948) 93-122.

5. Bouchard, L.S., Bronskill, M.J.: Magnetic resonance imaging of thermal coagulation effects in a phantom for calibrating thermal therapy devices. Med Phys. 27 (2000) 1141-5. 